\title{
Hypertrophic olivary degeneration and Purkinje cell degeneration in a case of long-standing head injury
}

\author{
JANICE R. ANDERSON AND C. S. TREIP
}

From the Department of Pathology, Tennis Court Road, Cambridge, and

The John Bonnet Clinical Laboratories, Addenbrooke's Hospital, Cambridge

SUMMARY A case is reported of a 52 year old man who sustained a head injury and survived for three years and two months in coma. He was subsequently shown to have severe brain stem damage, with a bilateral lesion of the central tegmental tract and bilateral hypertrophic olivary degeneration, associated with a widespread loss of Purkinje cells in the cerebellar cortex. Hypertrophic olivary degeneration is considered to be a trans-synaptic change associated with a lesion of the central tegmental tract, which in this case was thought to be due to primary shearing injury of the brain stem involving the superior cerebellar peduncles. It is suggested that, as a consequence of long survival, Purkinje cell degeneration represents further trans-synaptic changes, the result of neuronal degeneration in the hypertrophied inferior olivary nuclei and in the dentate nuclei. A chain or 'circuit' of degenerated neurones is thereby produced.

Hypertrophic degeneration of the inferior olivary nuclei is an unusual but well-recognized change associated with a lesion of the ipsilateral central tegmental tract or of the contralateral dentate nucleus of the cerebellum. In this condition, the inferior olivary nucleus appears swollen and the normal convolutions are obscured. It was originally suggested by Trelles (1944) that hypertrophic olivary degeneration occurred after a lesion of the dentato-olivary fibres passing to the brain stem, via the superior cerebellar peduncle, to join the contralateral central tegmental tract. Loss of Purkinje cells in the cerebellar cortex has not so far been reported in association with hypertrophic olivary degeneration. In this paper the finding of widespread loss of Purkinje cells associated with bilateral hypertrophic olivary degeneration in a case of long-standing head injury is reported.

\section{CASE REPORT}

A 52 year old right-handed man was involved in a road accident in 1967 in which he was thrown out of his car. On admission to Addenbrooke's Hospital 30 minutes after the accident, he was deeply unconscious and had apparently been so from the outset.
Examination at this time showed a flaccid quaß riplegia. The reflexes, however, were all very bris with bilateral extensor plantar responses. He showeeg decerebrate responses to painful stimuli. The pulse was 70 per minute and regular, the respiratory rate 20 per minute, and the blood pressure was 120/ $80 \mathrm{mmHg}$. A radiograph of the skull showed no fracture. There was a central calcified pineal gland. There was a double fracture of the right pubic ramus, but no other bony injury. The day after the accident exploratory burr holes showed the brain to be 'reasonably slack', with some cortical bruising at the right temporal burr hole. The right lateral ventricle was tapped and faintly blood-stained cerebrospinal fluid was obtained. A tracheostomy was performed and on the third day he was put on a respirator and positive pressure ventilation was maintained until the 10th day. Subsequently he survived for three years and two months after the accident and during this time never regained full consciousness and showed no significant improvement. He remained akinetic and mute, responding to painful stimuli, but not purposefully. He received an adequate protein diet, an anabolic steroid, vitamin and iron supplements. Ampicillin and colistin were used to treat chest and urinary infections.

Vertebral and carotid angiograms carried out two months after the accident showed that there was no obstruction of any major vessel and no space occupy- 


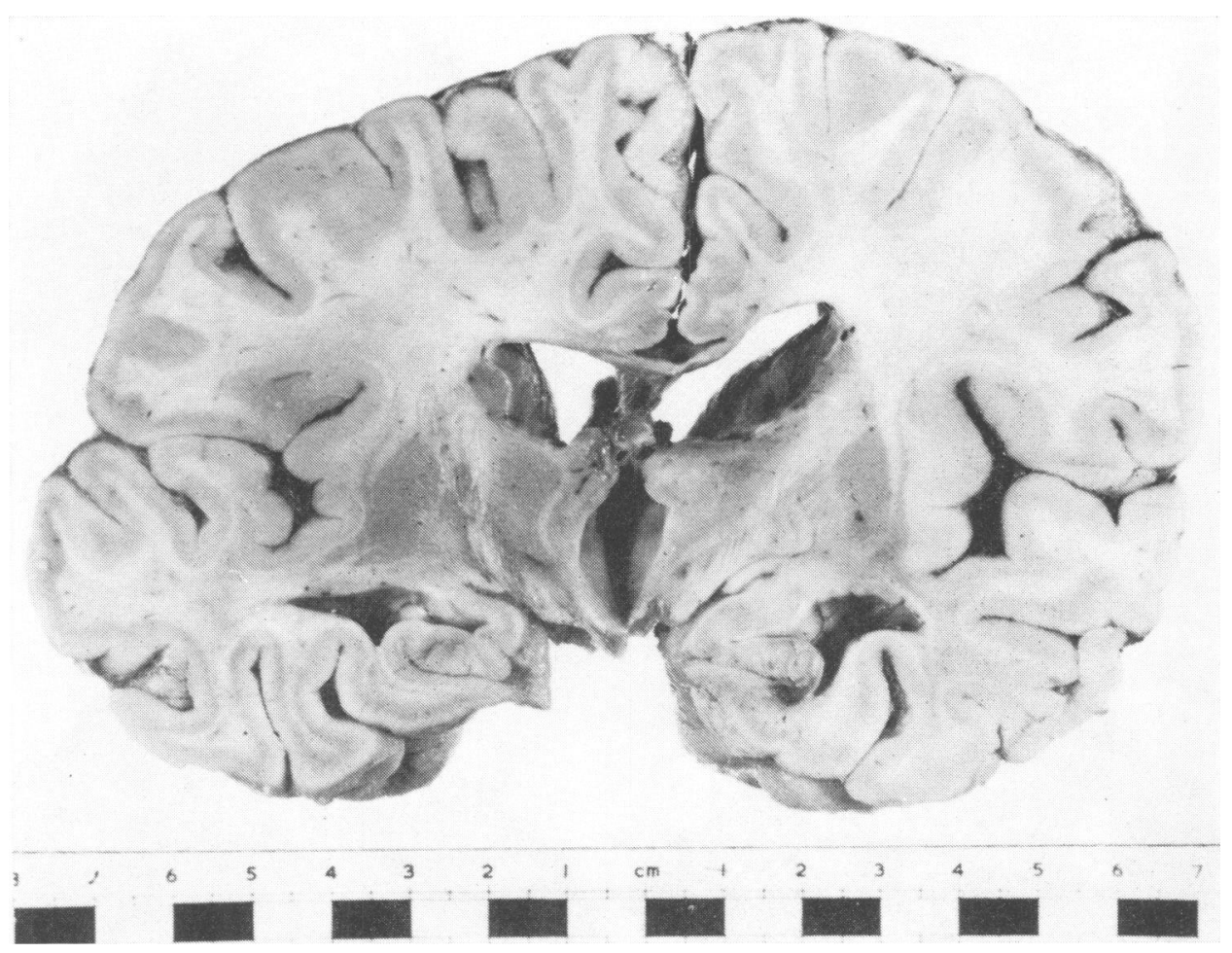

FIG. 1. Coronal section through cerebrum showing ventricular dilatation, widening of sulci, shrinkage of the left internal capsule, encysted haemorrhage in the right hippocampus, severe thinning of corpus callosum, old infarction of the fornix, and severe shrinkage of the mamillary bodies. $\times 4 / 5$.

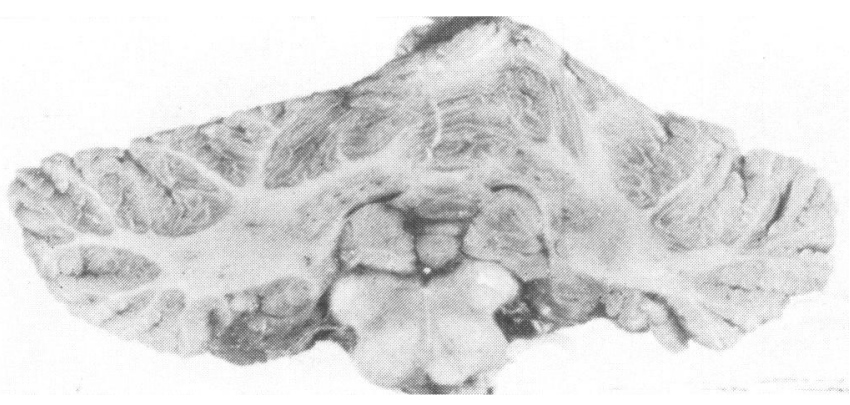

FIG. 2. Cerebellum and medulla. Atrophy of folia and prominent, white restiform bodies and inferior olives. The left pyramidal tract (on right of picture) is abnormally white. $\times 4 / 5$.

ing lesion. A pneumoencephalogram showed cerebral atrophy with striking enlargement of the third ventricle. Nine months after the accident, an isotope scan showed moderate external hydrocephalus for which a ventriculoatrial shunt was inserted, but this did not give rise to any improvement. He received the customary general nursing care for the unconscious patient, and three years and two months after the accident he was found dead, having been in his usual state 45 minutes earlier.

At necropsy the pathological findings outside the central nervous system were a bicuspid aortic valve, tracheal stenosis, and chronic pyelonephritis. Macroscopic examination of the brain revealed marked global atrophy of the cerebral hemispheres without external scarring. Dilatation of the ventricles, 


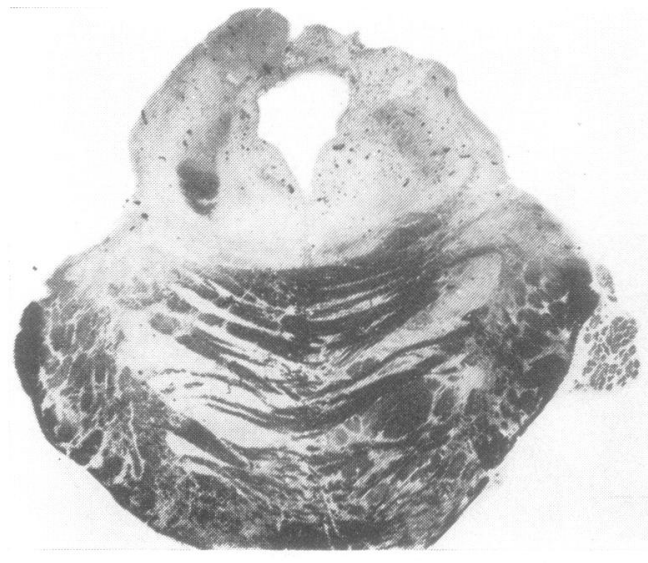

(a)

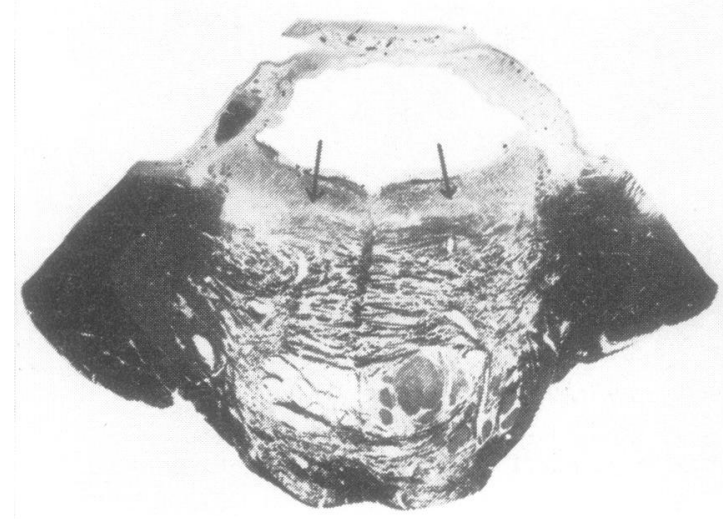

(c)

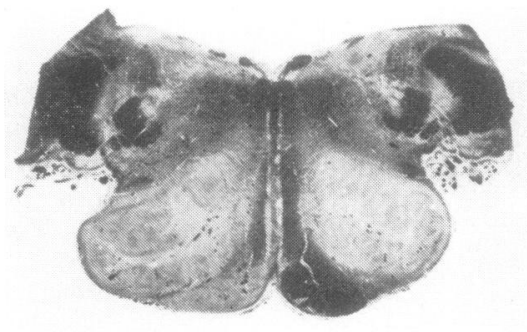

(e)

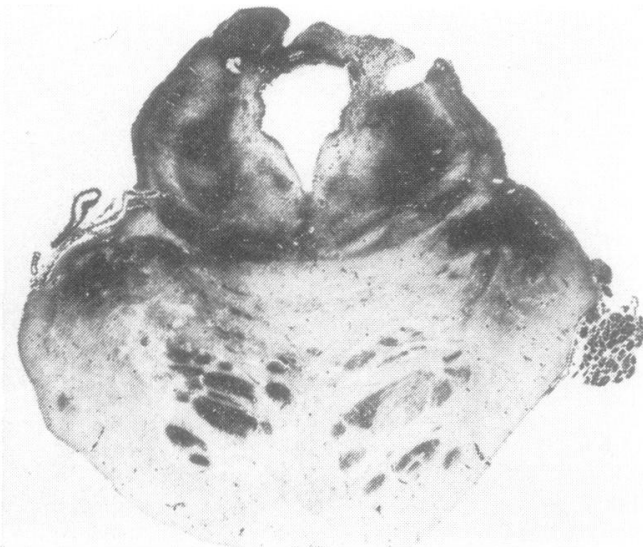

(b)

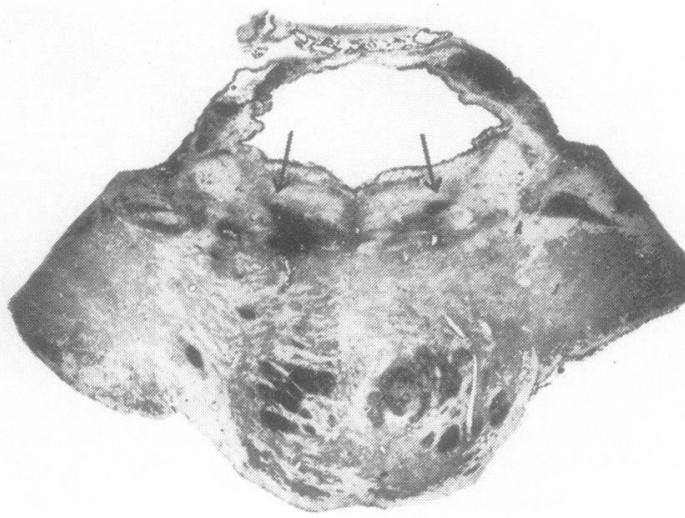

(d)

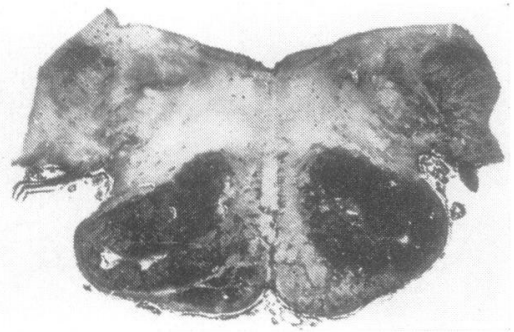

(f)

FIG. 3. (a) Rostral pons. Almost total myelin degeneration in both superior cerebellar peduncles (except for a음 few fibres remaining on the left), both medial lemnisci, central tegmental tracts, and corticospinal tracts, left $\gg$ more than right. Myelin, $\times 2$. (b) Rostral pons. Gliosis is shown in the areas of myelin degeneration. Holzer, $\times 2$. ․ㅡ. (c) Section through mid-pons showing myelin loss in the central tegmental tracts (arrows). Myelin, $\times 2$. (d) Mid-N pons. Gliosis in the central tegmental tracts, particularly the left (arrows). Holzer, $\times 2$. (e) Medulla. Symmetrical enlargement of olives with some flattening of convolutions. Pallor of medial lemnisci and left pyramid. $N$ Severe loss of olivocerebellar projection fibres. Myelin, $\times 2$. (f) Medulla. Severe gliosis of olives. Less severe $\mathbb{\omega}$ gliosis of restiform bodies and left pyramid. Holzer, $\times 2$. 


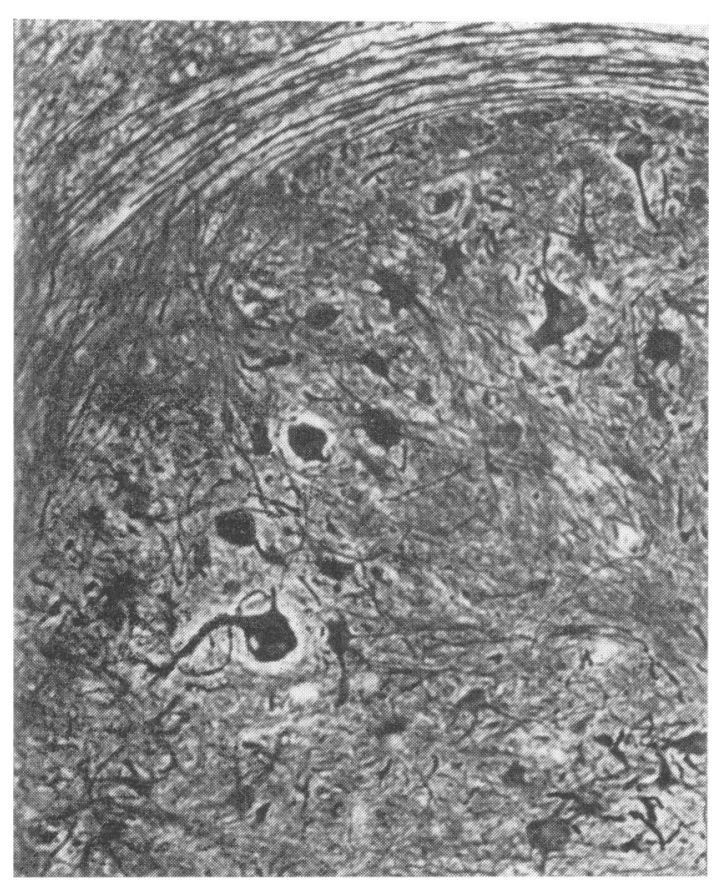

FIG. 4. Medulla. Enlarged, misshapen, and vacuolated olivary neurones. Palmgren. $\times 100$.

particularly of the third, was seen in coronal section. The left internal capsule appeared shrunken. The right hippocampus contained an old encysted haemorrhage. There was old infarction, with haemosiderin staining, of the fornix. The mamillary bodies were shrunken (Fig. 1). The quadrigeminal plate showed old infarction with yellow discolouration. There was shrinkage of the left ventral pons. There was moderate global atrophy of the cerebellar folia; the restiform bodies and inferior olives appeared white and sclerotic (Fig. 2).

HISTOLOGICAL EXAMINATION OF BRAIN Blocks were taken of most of the brain stem and cerebellum,

TABLE 1

NEURONE COUNT IN INFERIOR OLIVARY NUCLEI

\begin{tabular}{lc} 
& $\begin{array}{c}\text { Cells per high power field } \\
\text { (mean of } 10 \text { fields) }\end{array}$ \\
\hline Control & 16.4 \\
Left & 5.4 \\
Right & 6.4 \\
\hline
\end{tabular}

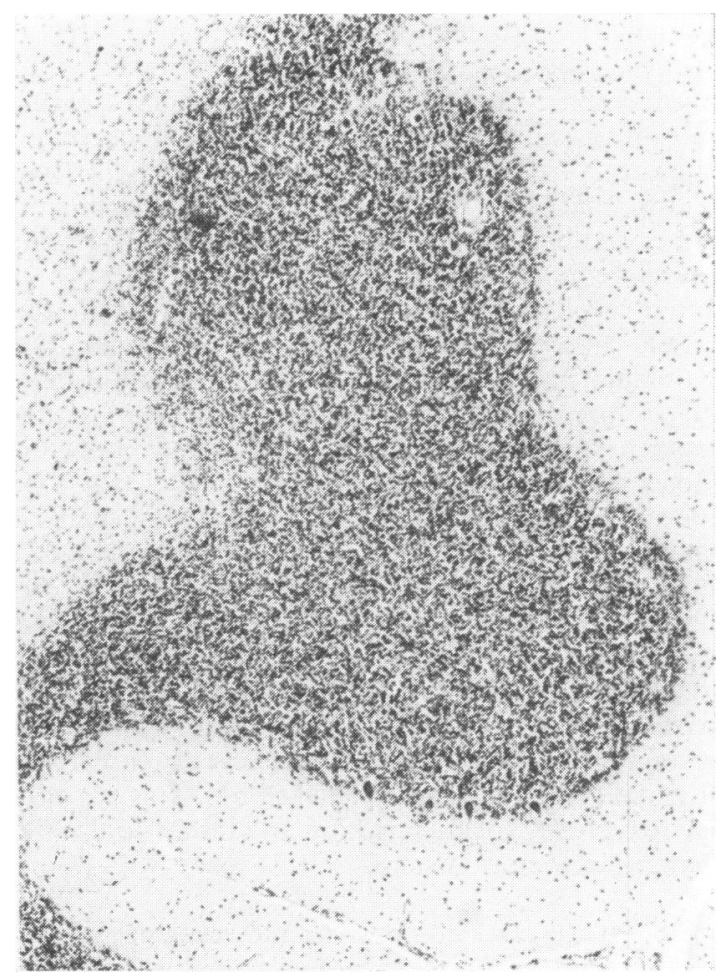

FIG. 5. Cerebellum. Severe loss of Purkinje cells. Nissl, $\times 30$.

embedded in paraffin wax and sectioned. Sections were stained with haematoxylin and eosin, cresyl violet, by Holzer's method for fibrous glia, by Palmgren's method for axons, and with iron haematoxylin for myelin. Frozen sections were stained for neutral lipids.

CEREBRAL HEMISPHERES Sections of representative areas of the cerebral cortex were histologically

TABLE 2

CELL COUNTS OF PURKINJE CELLS AND NEURONES OF DENTATE NUCLEI

\begin{tabular}{lcc}
\hline & Purkinie cells & Dentate nucleus \\
\cline { 2 - 3 } & $\begin{array}{c}\text { Cells per low power field } \\
\text { (mean of } 10 \text { fields) }\end{array}$ & $\begin{array}{c}\text { Cells per high power field } \\
\text { (mean of } 10 \text { fields) }\end{array}$ \\
\hline Control & $27 \cdot 9$ & $14 \cdot 1$ \\
Left & $12 \cdot 1$ & $12 \cdot 0$ \\
Right & $11 \cdot 1$ & $12 \cdot 5$ \\
\hline
\end{tabular}

Note: The reduction of Purkinje cells in the vermis was of the same order of magnitude as in the cerebellar hemispheres. 
normal. The underlying white matter, however, showed moderate fibrillary gliosis, together with considerable widening of the perivascular spaces, indicating some degree of long-standing atrophy. Evidence of continuing breakdown of myelin (free neutral lipid) was present in the corpus callosum and white matter immediately adjacent to it, but not elsewhere in the centrum ovale. Extensive gliosis and round-celled cuffing were also conspicuous in the corpus callosum and in the body of the fornix. The mamillary bodies showed neuronal loss and fibrillary gliosis, presumably degeneration secondary to the damage to the fornix. There was softening, with gliosis and haemosiderin deposition, of the left internal capsule at its junction with the cerebral peduncle. The hippocampus on both sides showed moderate gliosis, particularly of the end folia. Corresponding to the capsular lesion, there was degeneration and gliosis of the right lateral and left anterior corticospinal tracts in the spinal cord. All these changes were consistent with long-standing traumatic damage of moderate severity involving principally white matter (Strich, 1961). This paper is devoted chiefly to a description and discussion of the changes in the brain stem.

PONS In the rostral pons, at the level of the decussation of the superior cerebellar peduncles, there was degeneration of myelin in both superior cerebellar peduncles and in their crossing fibres. Some myelinated fibres remained in the decussation. Below the level of the decussation of the superior cerebellar peduncles (Figs $3 a$ and $3 b$ ) and in more caudal sections of the pons, there was almost complete degeneration of both superior cerebellar peduncles except for a few fibres remaining in the left peduncle. There was bilateral degeneration of the central tegmental tract. This was identified as an area of discrete gliosis, particularly well shown in the midpons (Figs 3c and 3d). There was prominent periaqueductal gliosis and severe replacement gliosis in the areas of myelin degeneration described above.

MEDULLA The inferior olives were symmetrically enlarged and had lost some of their normal convolutions (Fig. 3e). This impression was confirmed by the Holzer preparation (Fig. 3f), which showed dense gliosis of the amiculum. The neurones were reduced in number and were frequently abnormal, with bizarre shapes and vacuolation (Fig. 4). There was a striking loss of myelin and much gliosis in the hila of both olives, involving the olivocerebellar projection fibres. Some gliosis of the restiform bodies was present (Fig. 3f). Neurone counts were carried out on the inferior olives. The result is shown in Table 1.
CEREBELLUM There was widespread and severe loss $\stackrel{\mathbb{Q}}{\mathrm{C}}$ of Purkinje cells throughout the cerebellum (Fig. 5). The dentate nuclei also showed neuronal loss with $\stackrel{\text { ? }}{\subseteq}$ fibroglial proliferation. Cell counts of Purkinje cells ${ }^{\natural}$ in representative folia and of the neurones of the $D$ dentate nuclei gave the result shown in Table 2.

\section{DISCUSSION}

Hypertrophic degeneration of the inferior olivary nucleus is a change that may occur in association with a lesion of the ipsilateral central $\frac{\overline{\bar{c}}}{\frac{\mathrm{s}}{2}}$ tegmental tract or of the contralateral dentate $\stackrel{\mathbb{Q}}{\Omega}$ nucleus. It is not a change that has been reported after a lesion of the olivocerebellar fibres in $\vec{A}$ the inferior cerebellar peduncle. It is therefore considered to be trans-synaptic.

The characteristic features of hypertrophic olivary degeneration are overall enlargement of the olive, in which the convolutions are obscured. $\omega$ The neurones may appear large and deformed or and show vacuolation and there is a marked astroglial reaction. Gautier and Blackwoof (1961) demonstrated that there is enlargement of both grey and white matter and that the increas width of the grey lamina is in part due hypertrophy of nerve cell bodies and processes. They suggest that eventual atrophy of the abnormal neurones is not accompanied by shrinkage of the glial mass so that the enlarged outline of the olive persists.

Lapresle and Hamida (1970) showed that a localized lesion of the dentate nucleus is associated with a correspondingly localized $\stackrel{\odot}{\varnothing}$ hypertrophic degeneration of the contralateral $\cong$ inferior olive. They also reported two cases of $\overrightarrow{\overrightarrow{0}}$ vascular lesions in the region of the red nucleus, associated with hypertrophic olivary degeneration. They concluded that the dentato-olivary pathway passes through the superior cerebellar peduncle and, after crossing the midline at the decussation of the peduncle, passes beside the internal and dorsal surface of the red nucleus $\delta$ before joining the contralateral central tegmental tract and descending to synapse with the inferior 음 olive.

Hypertrophic olivary degeneration is a constant pathological finding in the clinical condition o of palatal myoclonus, but palatal myoclonus is not recorded in every case of hypertrophic $\stackrel{N}{N}$ olivary degeneration (van Bogaert and Bertrand, $\omega$ 


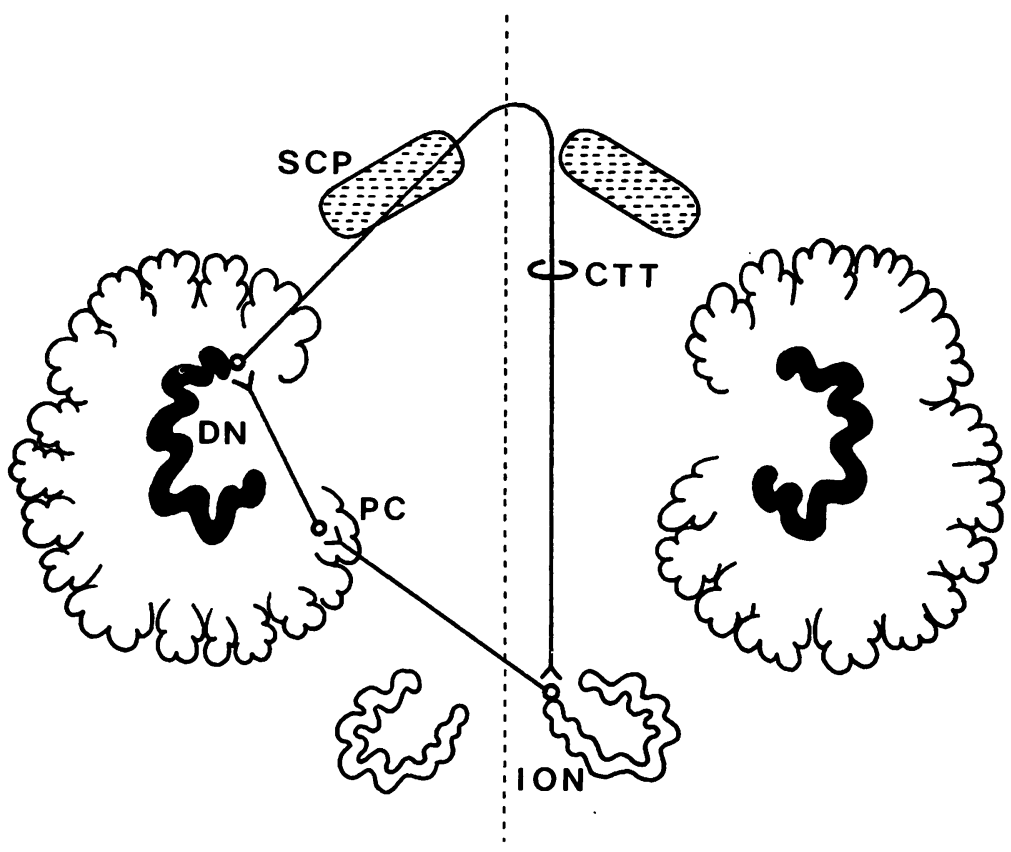

FIG. 6. Diagram to illustrate the 'circuit' of degeneration postulated in the text. CTT = Central tegmental tract. $\mathrm{DN}=$ Dentate nucleus. ION = Inferior olivary nucleus. $\mathbf{P C}=$ Purkinje cell. $\mathbf{S C P}=$ Superior cerebellar peduncle.

1928; Freeman, 1933). It was not observed in our case.

In this report, the changes seen in the inferior olives were characteristic of hypertrophic olivary degeneration, which in this case was almost certainly due to a bilateral lesion of the central tegmental tract. It seems probable that the myelin degeneration in the brain stem was the final result of primary shearing injury (Strich, 1961 ; Crompton, 1971). There was no record of raised intracranial pressure at any time in the clinical history and the clinical picture at the outset was suggestive of brain stem damage. It seems probable that the primary brain stem injury produced bilateral lesions, although shearing injuries are often characteristically unilateral (Strich, 1961). The region of greatest myelin degeneration is seen in the rostral pons, involving the superior cerebellar peduncle and the central tegmental tract on each side. There is a lesser degree of myelin degeneration in the decussation of the superior cerebellar peduncles, indicating that a single midline lesion is less probable. Nerve cell loss in the dentate nuclei, which was about $15 \%$ (see Table 2), is considered to be due to retrograde degeneration of the dentato- olivary fibres, secondary to lesions of the superior cerebellar peduncles.

There is no ready explanation of the severe and widespread Purkinje cell degeneration of the cerebellum, which involved both hemispheres and the vermis and was of the order of $60 \%$ (Table 2). Certain widely recognized causes of Purkinje cell loss must be considered: (1) Purkinje cell degeneration after profound hypotension can be eliminated in this case since there was no recorded episode of hypotension. Hypotension of sufficient severity to produce widespread Purkinje cell loss would moreover be expected to produce degeneration of cerebral cortical neurones (Brierley, Brown, and Meldrum, 1971) and this was not seen. (2) The familial cortical cerebellar atrophies and olivopontocerebellar degeneration can be eliminated as there is no suggestive familial or clinical history and the Purkinje cell loss, moreover, does not show the distribution characteristic of these conditions (Hall, Noad, and Latham, 1941). (3) There is no clinical history or pathological finding to implicate disorders such as heat stroke, chronic alcoholism, carcinomatous neuropathy, or lead intoxication, all of which may 
be associated with Purkinje cell degeneration.

It is suggested that, in this case, the Purkinje cell loss may represent a further trans-synaptic degeneration, as a consequence on the one hand of the bilateral hypertrophic olivary degeneration and on the other of degeneration of the dentate nuclei. Trans-synaptic degeneration is said to occur rarely in the adult nervous system; most authors, however, consider it to be the explanation of hypertrophic olivary degeneration. In the adult nervous system it is possible that degeneration may occur slowly in other nerve cells making synaptic contact with neurones that have degenerated (Blackwood et al., 1963). The climbing fibres that originate in the inferior olivary nucleus synapse directly with the Purkinje cells in the cerebellar cortex. In the present case, as a consequence of long survival after head injury, Purkinje cell degeneration may be due to prolonged deafferentation after hypertrophic olivary degeneration. In support of this suggestion, there is degeneration of the fibres arising from the hilum of the olive and gliosis in the cerebellar white matter, suggesting degeneration along the olivocerebellar pathway. The Purkinje cell degeneration could also to some extent be a retrograde process from neuronal loss in the dentate nuclei, as the Purkinje cells project to these nuclei. The dentate neuronal loss was, however, small $(15 \%)$, so that it could not account for more than a part of the Purkinje cell loss. There would, therefore, appear to be in this case a 'circuit' of degeneration involving the central tegmental tract, the inferior olivary nuclei, the Purkinje cells, and the dentate nuclei after primary damage of the superior cerebellar peduncle and tegmentum in the upper pons (Fig. 6).

We are grateful to Mr. John Gleave for permission to use the clinical records; and to Dr. Marion Smith and Professor P. M. Daniel for encouragement and advice. We are indebted to Mrs. Maureen Taylor for the histological preparations and to the Department of Medical Photography at Addenbrooke's Hospital, Cambridge.

\section{REFERENCES}

Blackwood, W., McMenemey, W. H., Meyer, A., Norman, R. M. and Russell, D. S. (1963). Greenfield's Neuropathology, pp. 19-20. Arnold: London.

Bogaert, L. van, and Bertrand, I. (1928). Sur les myoclonies associées synchronées et rythmiques par lésions en foyer du tronc cérébral. Revue Neurologique, 1, 202-214.

Brierley, J. B., Brown, A. W., and Meldrum, B. S. (1971). The nature and time course of the neuronal alterations resulting from oligaemia and hypoglycaemia in the brain of Macaca mulatta. Brain Research, 25, 483-499.

Crompton, M. R. (1971). Brainstem lesions due to close head injury. Lancet, 1, 669-673.

Freeman, W. (1933). Palatal myoclonus; report of 2 cas with necropsy. Archives of Neurology and Psychiatry, 29 742-754.

Gautier, J. C., and Blackwood, W. (1961). Enlargement of th inferior olivary nucleus in association with lesions of the central tegmental tract or dentate nucleus. Brain, 8 . 341-361.

Hall, B., Noad, K. B., and Latham, O. (1941). Familial cortical cerebellar atrophy. Brain, 64, 178-194.

Lapresle, J., and Hamida, M. B. (1970). The dentato-olivary pathway. Somatotopic relationship between the dentate nucleus and the contralateral inferior olive. Archives of Neurology, 22, 135-143.

Strich, S. J. (1961). Shearing of nerve fibres as a cause of brain damage due to head injury. Lancet, 2, 443-448.

Trelles, J. O. (1944). La oliva bulbar; su estructura, función y $\overrightarrow{\vec{F}}$ patologia. Revista de Neuro-Psiquiatría, 6, 432-521. 